\title{
Special volume on 'Methodological Advances in Queueing Theory' of the First ECQT Conference
}

\author{
Onno Boxma ${ }^{1}$. Joris Walraevens ${ }^{2}$
}

Received: 28 December 2015 / Published online: 21 January 2016

(C) Springer Science+Business Media New York 2016

The first European Conference on Queueing Theory (ECQT) took place in Ghent, Belgium, August 20-22, 2014. It continued a tradition started by the late Jesus Artalejo, who three times organized a Madrid Conference on Queueing Theory. ECQT featured about 80 presentations. The presenters of methodologically oriented studies were offered the opportunity to submit a paper based on their presented work to Queueing Systems. The 10 papers in this special issue have been chosen for publication after a careful refereeing process. We would like to take this opportunity to express our gratitude to the Editor-in-Chief Sergey Foss and the Springer staff for their advice, support and help. We are also indebted to the TPC of ECQT for aiding us in the review process and to the anonymous referees for their efforts. Below we give a brief overview of the accepted papers.

Avrachenkov, Morozov and Steyaert study multi-class multi-server queueing systems with retrials. Motivated by several telecommunication applications, they provide sufficient stability conditions. In some cases, these conditions are also shown to be necessary. Feinberg and Yang also consider a multi-server queue with several customer classes. They consider a pricing problem. The system incurs holding costs when there are customers waiting in the queue. An arrival joins the queue if the price of service is not higher than the maximum amount that the arrival is willing to pay, and this maximum amount depends on the customer type. The system manager wants to

\footnotetext{
$凶$ Onno Boxma

o.j.boxma@tue.nl

Joris Walraevens

Joris.Walraevens@UGent.be

1 Department of Mathematics and Computer Science, Eindhoven University of Technology, Eindhoven, The Netherlands

2 Department of Telecommunications and Information Processing, Ghent University, Ghent, Belgium
} 
choose the price, depending on the number of customers in the system. The authors describe average-reward optimal, canonical, bias optimal, and Blackwell optimal policies for this pricing problem. Chaudhry and Kim determine the distributions of queue length and waiting time for the $G I^{X} / M / c$ queueing model. They present a simple and effective derivation of the queue-length distribution at arrival instants as a linear combination of specific geometric terms involving the roots of the underlying characteristic equation. The main benefit of their method is that it is analytically simple, easy to implement and computationally efficient.

Badila and Resing study a two-queue coupled processor model which receives service requirements at both queues simultaneously. The service requirements at the first queue are always at least as large as those at the second queue, and if server 2 becomes idle, it starts to serve work from queue 1 (which is referred to as coupling). The ordering assumption allows the authors to relate their model to a queueing model of two parallel queues without coupling, and to provide an exact analysis of the joint workload distribution. Razumchik and Telek also are concerned with a two-queue model. In their case, a single server handles ordinary customers, but external resequencing signals arrive and move regular customers to a second queue with lower priority. The authors focus on the analysis of the delay of ordinary customers.

Abouee-Mehrizi and Baron analyse a state-dependent $M_{n} / G_{n} / 1$ queueing system. Both the arrival rate and the service rate may depend on the number of customers in the system, and the service rate can be modified at both arrival and departure instants. Focussing on the number of customers, the authors show that their state-dependent queueing system is equivalent with a Markovian birth-and-death process. The paper of Margolius and O'Reilly is devoted to a stochastic fluid model. This model is driven by an underlying continuous-time Markov chain with a time-varying generator $T(t)$ with the special feature that $T(t)$ is periodic with period one. They derive expressions for the key periodic measures for the analysis of the stochastic fluid queue, and they develop efficient methods for their numerical computation.

Fricker and Servel study a heterogeneous closed queueing network with $N$ singleserver queues and one infinite-server queue, as a model for a bike-sharing system. For large $N$, the limiting queue-length distribution is analytically studied by means of mean-field theory. A particular incentive policy to go to the least-loaded of two chosen single-server queues is also investigated. The paper of Jansen, Mandjes, De Turck and Wittevrongel considers an infinite-server system in a random environment: arrival rate, service requirements and server speed are modulated by a background process. Scaling both the arrival rates and the background process, a large deviations principle is derived for the number of jobs in the system. Lopatatzidis, De Bock, De Cooman, De Vuyst and Walraevens incorporate imprecise probabilities in the study of queues. For an elementary discrete-time queueing system, they assume probabilities of arrival and departure are only known to be in a given interval. For two different types of independence, they show how to calculate upper and lower bounds on various performance measures, such as the mean queue length. One of the main observations is that ergodicity may break down due to imprecision. 